\title{
Fetishising and Commodifying 'Training'?
}

\begin{abstract}
Chapter 3 examines the effectiveness of traditional capacitybuilding roles focused on the provision of training through Continuing Professional Development (CPD) or Continuing Medical Education. It draws on research evidence to expose the unintended consequences of interventions focused on forms of CPD 'training'. It describes the SVP approach favouring on-the-job co-working and mentoring over formal off-site courses. This approach increases opportunities for genuine learning and confidence in deploying new knowledge. More importantly, this reduces the collateral damage caused by traditional CPD interventions. Notwithstanding these 'successes', our research suggests that the effects of even these interventions can be short-lived. It was at this stage in the project journey that we realised that co-presence, whilst essential, was not sufficient to guarantee knowledge translation and sustained impact.
\end{abstract}

Keywords Continuing professional development . Continuing medical education · Conditionality

\section{INTRODUCTION}

Chapter 1 set out the objectives of capacity-building approaches to international development as exemplified in the Tropical Health and Education Trust's Health Partnership Scheme and the focus this places on knowledge leverage to support systems change. In this context, 
we have conceptualised the role of the professional volunteer as a 'knowledge intermediary'. Chapter 2 emphasised the critical importance of context to the effective placement of such knowledge intermediaries and the damaging unintended consequences that may arise from labour substitution roles. This logically implies a highly specified, structured and supported role for professional volunteers focused on (bi- or multilateral) knowledge mobilisation. Indeed, the risks associated with labour substitution may be entirely mitigated by a role delineation explicitly prohibiting clinical work. And, many 'free mover' short-term volunteers (outwith organised programmes such as the SVP) have effectively chosen this option in order to avoid working without clinical registration ${ }^{1}$ and professional indemnity insurance. In such cases, the desire to avoid complex legal and financial systems effectively drives the activity leading to a focus on fly-in-fly-out short courses.

Again, logically, these goals may imply a focus on initial (classroom) education taking place within the frame of education providers (colleges and universities). This would have the longer-term benefits of systematically training the next generation prior to their entry into the workforce. Deploying volunteers outside of health facilities, in formal (off-the-job) training encounters could protect them from the kinds of risks associated with clinical practice and increase their ability to focus on training. And, arguably, this approach, implying a focus on formal training, offers possibilities for substitution of physical presence with alternative (or complementary), environmentally responsible (low carbon) and cheaper modes of intervention. There are a number of reasons why professional volunteering, in the context of health partnerships, has tended not to focus primarily on initial education.

THET's Health Partnership Scheme (HPS) generally fosters or supports hospital-hospital relationships. In practice, this is less the case now than it used to be as universities and professional bodies (such as the Royal Colleges) are actively engaging in the HPS. Having said that, the pressures on organisations such as the Tropical Health and Education Trust to demonstrate value for money (and impact) have encouraged a focus on measurable short-term (in-out) interventions that are, at least in theory, amenable to metrics. THET fully acknowledges the importance of long-term relationships and the problems of achieving and capturing change in the short term. However, as with all funding bodies, project time frames are usually very short (20 months, for example) and THET are under pressure to demonstrate impact within these time frames. 
Initial (diploma or degree-level education) is a longer-term investment and offers less potential for measuring specific and attributable systemic outcomes.

A second factor relates to the supply of professional volunteers. The majority of professional volunteers are motivated, at least in part, by their personal needs for learning and 'mobility / career capital'. International exposure has increasingly become a 'rite of passage' in medicine and other careers. Non-clinical placements are of little interest to many career track professional volunteers (Chatwin et al. 2016). As with funding bodies, professional volunteers are very keen to see change within the time frame and, wherever possible, directly attributable to their own activities. Whilst cautious progressive and collaborative incrementalism may be the most appropriate and effective mechanism for change in health systems, this approach may not appeal to funding bodies or volunteers.

Finally, and perhaps most importantly, it is widely accepted that education and training requires more than formal classroom teaching. Whilst classroom teaching may form a critical element of theoretical/ explicit knowledge acquisition, ensuring the learning of more tacit knowledge and implementation skills requires co-present learning 'onthe-job' through supervised placements and mentoring. The emphasis on 'lifelong' learning in the UK also places firm emphasis on structured continuing professional development to update and reinforce learning. This is the regime that professional volunteers and professional organisations (including the Royal Colleges) are accustomed to and therefore seek to replicate in Uganda. In other words, CPD is part of the culture of professionalism in the UK linked to professional development review (PDR). And, in that context, it does not translate, literally, into a more general interest in lifelong learning but implies short courses and an almost box-ticking exercise.

Chapter 3 responds to the following questions;

- What do you do with co-presence when you have achieved it?

- How do you deploy professional volunteers optimally as knowledge intermediaries?

- How do we encourage systems change relevant learning in this environment? Or, put more simply:

$\circ$ Who do we train?

- What do we train?

$\circ$ How do we train? 


\section{The Perceived Need for Continuing Professional DeVElopment IN LMICs}

There can be no doubt that there is a need for continuing professional development (CPD) or Continuing Medical Education (CME) in the Ugandan health system. This is not the same thing as saying that Uganda lacks the capacity to train its own health workers. The main need for CMEs and the one that is immediately observed by volunteers when they arrive is that the majority of staff they directly encounter on the wards dealing directly with patients and typically unsupervised are at junior level. Some hospitals depend almost entirely indeed on (unsupervised) student nurses and intern doctors to deliver services.

As we have noted in Chapter 2, this does not mean that there are no or even few very highly skilled and competent clinicians in Uganda but it is rare to find them on public wards or supervising/mentoring those staff who are. During a short (two week) visit, a senior British obstetrician working on one of the busy obstetric theatres in Mulago Hospital expressed dismay at his initial experience referring to the 'sheer butchery' he had witnessed. It was only later that he was made aware that all of the doctors in theatre were much junior interns with little experience or supervision. Add to this the overwhelming congestion and sense of chaos made worse by the absence of effective patient management systems (and triage) and it is not surprising that volunteers identify an immediate need for training. And perhaps this impression - of abject need - and absolute lack of resource - is functional in terms of attracting AID. On that basis, local managers may hesitate to challenge this naïve prognosis.

This situation has led to an emphasis in health partnership interventions on CPD or CME, a term used more commonly in Uganda and perhaps reflecting once again the dominance of the medical paradigm. Another factor reinforcing this emphasis on short-course interventions concerns the emphasis within internal (project) evaluation processes on easily obtainable metrics. The following caption frequently displayed by THET to promote the HP scheme indicates the emphasis on training and the challenges of trying to capture more holistic impacts on human resource management systems. The data presented here capture the easiest metrics: numbers of people trained. And measuring this is far easier if staff are taken off the wards and put into rooms where they can be counted. Unfortunately, this tells us nothing about the effectiveness of interventions and their impact on health systems (Fig. 3.1). 


\section{THET AT A GLANCE}
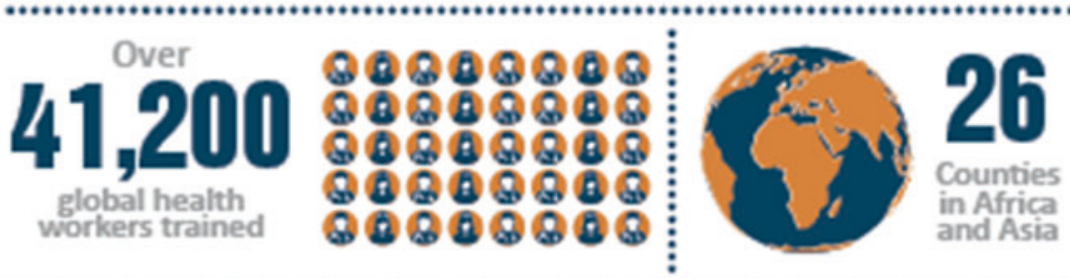

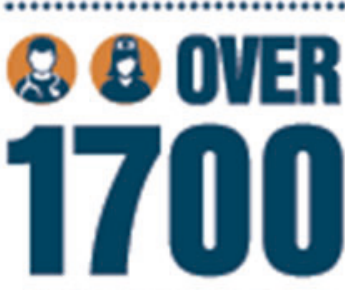

NHS health workers have volunteered with projects
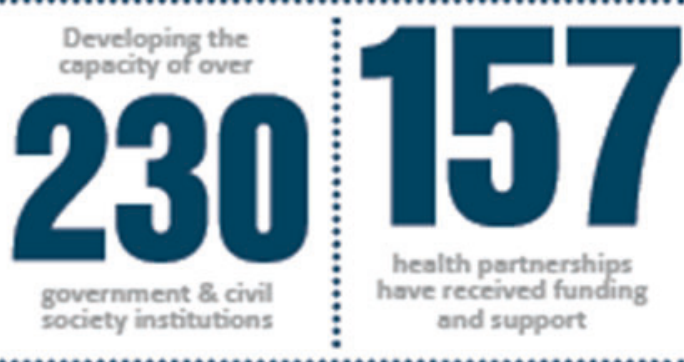

HEALTH SPECIALTIES

- Maternal and Newborn Health

- Non-Communicable Discases (NCDs)

- Sexual and Reproductive Health

- Palliative Care

- Accident and Emergency

- Surgery

- HIV/ AIDS, TB \& Malaria

- Medical Engineering

- Child Health

- Eye Health

- Mental Health

- Occupational Therapy

- Blood supply

- General Health

Fig. 3.1 Numbers of staff trained in the health partnership scheme (Source: THET 2015)

Having said that, we fully understand the challenges facing organisations like THET under pressure from their own paymasters (DFID) and seeking to justify the expenditure of public funds on development interventions in a time of austerity and swinging cuts in public services. Not only is this form of AID 'hard to get right', as Bolton (2007) suggests, but it is also incredibly difficult to capture. And providing the evidence base demands highly sophisticated and time-consuming approaches which fail 
to sit easily alongside the 'smash-and grab' demand for 'objective' (read quantitative) proxy outcome indicators.

The dominant approach to improving care [in low-income countries] (as a result) involves continuing professional development but (as Byrne-Davis et al. point out), 'little is known about their impact on practice' (2016: 59). One might argue that the best way to deal with this situation is not to deploy British professionals as volunteers but to improve local human resource management systems to require senior staff to be present and take on training roles, and for the health system itself to develop CME systems rather than relying on foreign interventions. In the capacity-building work we are currently engaged in, in the area of bio-medical engineering aimed at increasing the skills levels of practising technicians, we are beginning to lobby for the development of a CME system and to support the development of its constituent modules. At present, no such thing is in place. $^{2}$

However, for the time being, this is the context within which professional volunteers will engage on a day-to-day basis. One might argue that training in itself is innocuous and can only add value. And, the more people we train presumably the more potential there is for positive systems change. One of the SVP volunteers expressed this view in an interview and in response to a question about the role that volunteers could play in system change:

I don't even know how [a volunteer] would go about [engaging with systems]. There are so many levels of mistrust and corruption. Where to even start to infiltrate the system and go about it in the right way especially if you're a foreigner trying to come in and introduce policy. It's not even a can of worms. It's like a reservoir of snakes. Education is something you can't take away from someone and so that's something we can do continually, teach and set an example and then it's up to that individual if they carry on what you have imparted.

As the respondent notes - this approach is aimed primarily at individual clinicians and not systems as such. To the extent that a system is the sum of its constituent parts, then training individuals may in the very long term have a systems impact. Or, the individuals could become so demotivated (threatened even) when trying to utilise these skills on an individual basis that they either give up or leave the system. 
Certainly it is essential that deploying organisations (including foreign NGOs) adopt some humility and accept outright that they cannot begin to train all the health workers in Uganda and, as such, this individual ('drop-in-the-ocean') approach is unlikely to generate systems impact.

\section{The Commodification of Training: Short Courses as Personal Income}

In high-income countries, training is unashamedly commodified: CMEs are a commodity that individuals (or their employers) often pay a very high price for. And, failure to engage in these forms of portfolio training linked directly to compulsory and structured professional development review (PDR) have an immediate and serious career impact. In that respect, treating training as a marketable commodity is not novel. The commodification process in LMICs rather turns this on its head. In this situation, health workers expect to be paid in order to train.

In practice, we have identified some serious unintended consequences associated with formal training. It has been traditional in Uganda and is now quite expected that CMEs delivered by foreign NGOs take place off-site in hotels or educational facilities. This has two immediate effects. First, it takes staff off clinical duties in an environment when wards are already barely staffed. And there is no culture or system for providing staff cover. It is not at all unusual when we find no staff present to be told that they are 'on a course' as if training can ever be a justification for leaving neonates unattended. Referring to a recent visit to a low-resource setting, a colleague at a meeting on global health in the UK reported:

It was the most depressing visit I've undertaken - all key staff were away on courses elsewhere.

Secondly, people attending these CMEs generally expect to be paid a 'per diem' (daily top-up) plus expenses. In an environment where wages are so pitifully low, CMEs have become a precious and competitive commodity. It goes without saying that foreign NGOs are entirely responsible for this situation, creating, as Moyo predicts (2009), new opportunities for absenteeism and corruption. 


\section{Who Are We Training: 'We Need Some Motivation'}

This request, made to us repeatedly, was at first taken at face value to imply, literally, that staff felt deflated and low in spirits, given their levels of remuneration and working conditions. It soon became clear that the verb 'motivation' is being used rather differently, as a noun, to ask for money.

It is interesting that Mathauer and Imhoff explain how, in Benin, the term 'motivation allowances' is used to describe financial incentives to attend training and go on to explain how that has 'changed the meaning of motivation from a state of mind to that of an incentive $[\ldots]$ giving the word a new meaning' (2006: 6). In effect, this has devalued training as an investment in its own right and created perverse incentives to lobby for and attend formal training.

A focus group involving three Ugandan health workers who have worked alongside many SVP volunteers expressed a similar experience when asked about the progress of SVP involvement in their facility:

What I will talk about is the motivation because the times I have been with health workers going to workshops, it is a case in Uganda if they go for a workshop they think they will get a transport refund or per diem. I think that's what has made it not go on very well. Because they think your [SVP] volunteers come for training, you just train them but they will not get any per diem so they tend not to attend the workshop. ${ }^{3}$ I think it doesn't work very well just because most people in the public sector think of money. The health workers in the public sector think of money. That's what I have seen. Sometimes the [SVP] volunteers would organise CMEs but most of the staff were talking about money. Are they going to give us some money? So most of them did not attend. Or if they happen to attend they come in for 30mins and see if they are signing [for expenses] and if there isn't [signing] they will walk away. Others come at the end of the session [just to sign and claim expenses] (FG).

His colleague then asks us:

How does that affect the volunteers and people like you? Because ultimately the volunteers want to train people so that they can work more effectively in adverse conditions. You know you [SVP] are doing a lot of jobs and you work long hours. How can you benefit the trainings the volunteers offer if people bave such a mind-set? How will that shape the relationship with the volunteers and people like you? 
[One of the participants responds] I think we should tell people we don't have any money to give. Yes, they should tell them that - there is always the expectation of money (FG)

In another context, a workshop on placements involving a team of UK health trainers discussed frustrated attempts to achieve attendance at CMEs in Uganda and the constant pressure to pay per diems. One of the participants who had visited a Ugandan health facility on many occasions to do training stated:

We are told we have to take into account their passivity but it isn't that they are passive; it is that basically they don't want it (the training). Facilitation would help. If we put $£ 62 \mathrm{k}$ behind it they would love the education but we are where we are. If we don't pay facilitation they won't come.

Other participants at this policy meeting agreed and suggested that paying per diems could be a solution to attendance problems. As participants in the meeting ourselves we urged them not to fall foul of the pressure to pay people for attending training. In the medium term this does nothing to encourage learning over and above ensuring that there are 'bums on seats' (trainees to be counted).

The distorting effects of per diems also have the effect of denying training to many clinically active health workers so that the same people - often those seeking to avoid clinical work - attend repeated training. Mathauer and Imhoff, again in the context of Benin, emphasise not only the wasted opportunity but also the tensions this can cause within staff teams:

Opportunities for training must be equitably allocated. They should not always privilege the same people (2006: 11).

The immediate association of CMEs and 'projects' with income colours relationships creating not only expectations of volunteers (in one case a UK doctor was referred to as 'Dr Donor') but also fomenting jealousies and suspicions. The following quotes from Ugandan health workers and cited in our Policy Report on 'Volunteer and Health Worker Relationships' (Ackers 2014) are typical:

When projects get involved everyone assumes that someone is getting paid (by the project) - that there are backhanders going on - so we need to be very accountable. 
Interestingly the word 'project' in Uganda has its own quite specific meaning connected to large income streams and often US AID. 'Project' for many health workers simply means income stream.

There is this mentality I'm sorry to say - that if you are associated with white people they think you are getting something in the office ... people need to be explained to, 'I am here to work. I am not paying people. I am here to exchange knowledge'.

Equity is an important issue and it is essential that clinically active frontline public health workers are immediately engaged in training. It is not always the case that senior managers (often doctors or clinical officers who refuse to engage in clinical duties in the public sector) attend the training. In some cases, SVP short courses have attracted staff cadres for whom the course has little direct relevance and where there is little, if any, chance that the skills will subsequently be utilised to improve patient services. Table 5 gives just a few examples of the cadres of students who presented themselves for a short course on emergency obstetric skills run by SVP volunteers. In this case, University staff organised attendance. One of the participants showing the greatest improvement in knowledge in post-training tests was actually a pharmacist. In the evaluation report, he noted that the 'main barrier' to using the obstetric skills was the fact that he was indeed a pharmacist.

Table 3.1 Before and after test results on a CME on emergency obstetric care

\begin{tabular}{lcc}
\hline & Before & After \\
\hline Nursing student & 7 & 16 \\
Midwife & 13 & 17 \\
Nursing officer/registered midwife & 14 & 16 \\
Nurse & 10 & 16 \\
SHO doctor & 14 & 18 \\
Ophthalmic nurse & 12 & 12 \\
Intern pharmacist & 5 & 14 \\
Public health student & 8 & 18 \\
Intern nurse & 15 & 17 \\
Nursing student & 6 & 13 \\
\hline
\end{tabular}

Source: SVP data 
In June 2015, the authors held a workshop in Fort Portal on human resource management in the public health sector inviting what we hoped would be the in-charges of all local facilities. At first we were a little surprised and even disappointed that very few of the in-charges came even though the District Health Officer and the Secretary for Health in the Region were addressing the event. However, the final session, which focused on gauging the views of the audience, made us realise some of the reasons why the target audience (more senior people) had not attended and the real benefits of this in terms of providing a unique opportunity for clinically active health workers themselves to attend an event and express their views. It transpired that a conflicting event ${ }^{4}$ was being held in the town sponsored by an American NGO which provided generous per diems. These opportunities were then immediately taken up by the senior staff leaving scope for others to attend our event which provided a meal and local transport costs only.

Asked in the final session to identify their main concerns in terms of HR management, delegates immediately spoke about what they called 'delegation'. At first we found this concept hard to understand until they explained that they meant delegation of opportunities to attend events and CMEs:

Delegation - somehow it is a problem in our health facilities - picking up knowledge is the most important thing about coming to these (training/ knowledge exchange) days. [Managers] are not delegating to lower level staff - the in-charges are going on all the CMEs and they are not sharing the knowledge when they come back to the facility. So, for example, they may learn about hand washing but they are not sharing it so it is not becoming practice. The problem is the same people are going to all the seminars every week; others are not given opportunities: 'the work of my staff is to work and the work of the in-charge is to attend training'. The others are not getting access to the knowledge so it isn't affecting service delivery. Management can't delegate - this is the challenge (Participant 1 )

My in-charge goes on one workshop then the next week he goes to another and another - there is no way of implementing the learning and no way that other colleagues can go. It is unfortunate the DHO [District Health Officer] has gone [left the meeting]. If the in-charge has attended a seminar 5 times then they should send other people - this is what Baylor [a US NGO] is now doing (Participant 2)

They attend too many workshops - the same workshop over and over again - they have not time to delegate or implement their learning (Participant 3) 
One of the speakers suggested managers may need training in the art of delegation but it became clear that this was much an issue of personal income as lack of training:

One of the problems is the implementing partners - the big NGOs organise many workshops and pay huge allowances so they all rush to those workshops and when there is a workshop where there is no money - everyone laughs - then, the point is - we get invited. If the in-charges get invited and there is no money they will delegate. Surprisingly the in-charges have communication with the organisers of the workshops and they know how much they will get paid- one million shillings etc. - so in fact we should make all workshops so people come here and pick knowledge without getting any money - they come away only with knowledge not money. If you got back with that knowledge and maybe a meal but no money you will go back with that knowledge and want to implement it.

One participant then suggested that, 'we should go back to all NGOs etc. and propose standardisation of per diems.'

These comments emphasise the damaging effects that foreign engagement, even in such an apparently innocuous area as CMEs, can have and the importance of behaving responsibly and collectively. A study of the perceptions of per diems in the health sector in Malawi and Uganda (Vian et al.) came to remarkably similar conclusions that per diems resulted in unnecessary trainings, caused conflict, contributed to negative organisational cultures, fraud and 'were perceived to provide unfair financial advantages to already better-off and well-connected staff' (2013: 237).

Corruption is highly entrepreneurial and the incentives provided for training are a prime focus of such 'innovation' designed to extract personal gains from foreign partners and volunteers. A recent exchange involving a request for short course training for drivers of a motorcycle ambulance in Uganda is illustrative. In this case, we were requesting support from an HUB partner. The Ugandan lead willingly agreed to 'loan' us one their drivers trained by the Health Partnership for one week. When it came to payments we were taken aback by a request for us to 'provide his per diem of $150,000 /=$ per day for seven days'. This equates to a monthly salary of over 4,000,000 Ugandan shillings - ten times what a midwife gets paid. In this case we have learnt that such payments do not go to the individuals but involve a major top-slice (cut) for a number of intermediaries. Training becomes a lucrative 
gravy train and 'donors' are made to look like fools. This provides a very valuable example of how localised tacit knowledge trumps the apparent expert knowledge of outsiders; a point we return to in Chapter 5.

In conclusion, this section has shown how short courses have effectively become 'cash-cows' valued for the financial incentives typically associated with them. This has the damaging externality effect of restricting access to those health workers who most need training and reducing the prospect that training is used or shared. In response to our emerging understanding of this local context, the SVP has progressively moved away from any association with per diems and has actively campaigned to discourage the per diem culture. We have also encouraged the practice of holding CMEs as close as possible to the health workers' workplace to ensure contextual relevance and reduce the losses to clinical work. This has extended to active infrastructural work to provide high-quality training facilities proximate to hospitals and health centres.

The next section returns to a point made before, about the role of foreign intervention in CPD training. In the UK, CPD is viewed as a system with opportunities for review and progression and within the frame of workload management. It is not simply a case of doing random courses as and when they arise. This is not the case in Uganda. The view that all training is good coupled with the received wisdom that attendance at training (even if only to 'sign') provides a legitimate reason for absenting oneself from work coupled with personal financial gain results in a high demand for training. On arrival, a professional volunteer or organisation will immediately perceive this as a thirst for knowledge and respond accordingly.

Arguably, training needs to be valued as a commodity as it is in the UK, not to generate profits for training providers but to render training sustainable. The following quote from a leading member of the $\mathrm{MOH}$ in Uganda explains the dilemma:

[We have] reservations about funded programs because usually when the funding runs out, the life drains out too. Free trainings which are entirely dependent on funding are not self-sustaining, however, they can be sustained if the trainings are at a fee (minutes of meeting)

Notwithstanding the problems outlined earlier, CMEs undoubtedly constitute one of a range of potential knowledge transfer (often fairly 
unilateral) mechanisms. In that context, the next section considers the content of the training (curriculum issues).

\section{What Do We Train (In CMEs)?}

When we first became involved in volunteer deployment in Uganda, one of the needs identified by UK doctors, perhaps fuelled by their initial exposure to clinical practice on the wards, was for 'emergency obstetric training.' And, the response was to develop short courses often based on established UK protocols with some adaptation to meet the rather different needs of a low-resource setting. At face value there can be no apparent problem with this. However, we became aware quite quickly of two concerns. First, that in an environment like the National Referral Hospital, any number of well-meaning NGOs from across the world could be devising such courses, based on their own national schemes and delivering them, often consecutively but on occasion simultaneously and often with the same cohort of staff (as noted earlier). A senior manager in Uganda made the following statement in a letter about CME input by international volunteers:

We are happy to welcome colleagues from overseas but we need to ensure that their contribution is carefully evaluated, communicated and coordinated ... to streamline the system. This will add significant value to the sum of international development effort and enable us to build sustainable collaborations.

Present practices not only result in duplication but worse still, confusion, as the participants are unsure - when information conflicts - which approach to use. We also encountered a surprising degree of defensiveness and territorial behaviour on the part of foreign clinicians reluctant often to compromise on specifics when a more simple back-to-basics approach would have been far more useful in that context. And, secondly, following communication with the relevant Ugandan professional bodies, we found that there were national CME programmes in existence and being delivered in areas such as emergency obstetric care. The Ugandan Association of Obstetricians, for example, was actively using an adaptation of the Canadian ALARM course, as the basis for CMEs using obstetricians and midwives trained in the use of that programme. SVP volunteers suggested to us that these programmes were fit for purpose although senior clinicians 
working with international NGOs continue to argue over finer details restricting the potential for standardisation and local ownership.

Similar problems have emerged where foreign NGOs have intervened to teach neonatal resuscitation using their own protocols and found themselves overlapping with the Ugandan adopted 'Helping Babies Breathe' programme.

This duplication is certainly a major problem in contexts such as the National Referral Hospitals and other large facilities overwhelmed with NGOs. In other more remote areas, health workers may have received little attention from international NGOs. However, in practice, NGOs do venture to many places often 'imposing' (with the best intentions) their training schemes. Whilst communication with senior gatekeepers (such as the District Health Offices ${ }^{5}$ ) is often obligatory more active discussion with the relevant people on the ground and within the facilities is unusual.

It was interesting to meet two clinicians from a US NGO at a very small Health Centre III facility that we have been actively working in recently, setting up a training scheme for laboratory workers completely unaware that we had recently refurbished the laboratory and local staff were managing testing very effectively. One factor that tends to exacerbate this problem is the tendency of local managers not to advise NGOs about other actors on the scene in order to optimise engagement and opportunities for top-up payments and per diems. A British medical volunteer who contacted the SVP to discuss her potential involvement in training was clearly aware of this problem:

I am very aware that when one visits these hospitals they are full of enthusiasm and make the visiting team feel not only welcome, but as if they are the only people helping. I have heard from other sources that both the Lifebox training and possibly the Safe Obstetrics course has already been delivered in [town], but apart from the WHO checklist in theatre, which is not used and a couple of lifebox oximeters, the staff deny all knowledge!!

An international conference on Healthcare Collaboration in Uganda held in Canada in 2014 (with very active Ugandan participation ${ }^{6}$ ) highlighted the need for improved communication and collaboration between external teams and with Ugandan leaders to encourage consistency in training; ensure awareness of the purpose of professional volunteers and promote mutual goal setting. A follow-up meeting between Professor Ackers and 
the Deputy Director of Mulago Hospital suggested that professional volunteers engaging in maternal health work were failing to work together, often generating confusion, restricting the effective transfer and application of knowledge and skills through unnecessary duplication and contradiction. Feedback from the SVP evaluation indicates parallel concerns among professional volunteers. In some cases, international relationships blossom into fruitful co-working. In others, volunteers are faced with unexpected and at times unwelcoming/competitive co-presence with other international volunteers.

\section{What do People Learn from Short Courses?}

We know relatively little about the impact that CMEs have on health systems in LMICs. This is not the same as saying people do not learn much but evidence that learning translates into changes in clinical practice, which in turn improve patient well-being and outcomes is elusive. One approach we have been encouraged to use in the SVP, more as the basis for external evaluation for THET, but also replicating approaches used in UK CMEs, is the practice of pre- and post-testing. These tests typically take the form of a simple multiple choice questionnaire focused on explicit clinical/technical skills. The tests are quite useful in capturing immediate (and explicitly clinical) knowledge acquisition and identifying areas where healthcare workers need further support.

\section{The SVP Emergency Obstetric and Neonatal Care (EMONC) Training Course}

SVP volunteers, in common with many others, were keen to engage in short course training in the area of EmONC. A short report (Tate 2014) describes our intervention in this area, which was integrated within an on-going mentoring and co-working programme. It was designed by an experienced British clinician who has worked for many years in Uganda and other African countries as part of the Liverpool School of Tropical Medicine 'Making it Happen' programme. In order to avoid taking staff off wards for too long, the SVP course took the form of an intense 2-day programme using 
Table 3.2 Pre- and post-test results

\begin{tabular}{lcc}
\hline Role & Pre-course (\%) & Post-course (\%) \\
\hline Doctors & 77.5 & 87.5 \\
Registered midwives & 58.5 & 83.5 \\
Enrolled midwives & 62.5 & 81.5 \\
\hline
\end{tabular}

Source: Tate (2014: 7)

mannequins in practical stations to encourage hands-on learning. We also used the conference room that we developed with a tent on hospital premises. The curriculum is focused on clinical skills including, for example, neonatal resuscitation, observation and early warning scores, management of eclampsia, sepsis and haemorrhage. A pre- and post-course questionnaire comprised 20 true/false questions including the following:

1. Low blood pressure is an early sign in haemorrhage $\mathrm{T} / \mathrm{F}$

2. Intravenous fluid should be given at a rate of 1 litre every 2 hours in hypovolemic shock $\mathrm{T} / \mathrm{F}$

3. Raised respiratory rate is a sensitive measure in shock $\mathrm{T} / \mathrm{F}$

4. In septic shock, patients should be given fluids at a rate of 1 litre over 20 minutes $\mathrm{T} / \mathrm{F}$

The tests results showed improvement in all of the participant's postcourse knowledge (Table 3.2):

In this case, given the ongoing co-presence of volunteers on the wards, we were able to gain some feedback on the immediate impact of the training on staff. Volunteers noted improvements in their behaviour and practice:

The midwives have shown improvement in their clinical practice and drug knowledge, as well as spotting and managing obstetric emergencies (V).

Working in Mulago Hospital with a junior doctor who had attended, a volunteer noted that observations were being taken with greater care and with closer attention to detail: 
The interns were also sharing their improved knowledge with other interns on the ward, it was received with interest and enthusiasm and was actively being used in clinical practice. One recommended: The confidence with the improved knowledge was infectious (V).

A midwife participant also reported:

A few days after the course I had a patient overnight with severe PET, I felt I managed it better than I would have previously, I was confident to manage instead of referring (UHW).

On the basis of this experience we can assert, with some confidence, that the teaching is translating into relevant learning and that this is shaping individual practice and, on occasions, being shared with peers. The courses also provided opportunities to identify skills areas that prove a particular challenge to health workers. In one case, for example, an SVP neonatal nurse developed a CME programme involving short (2-hour) courses every week over a 6-week period (again delivered proximately on site). The test results showed overall improvement in knowledge with some significant weakness in their ability to understanding the mathematics behind dilution of medication for neonates. This enabled her to do further work on this area. It is difficult to say whether the staff later improved their practice mainly because the majority were not working on the neonatal unit and those who did were rotated out of it or left on a regular basis. Only one of those trained continued to work with our volunteer after the course ended. Staff rotation remains a persistent barrier to achieving any critical mass of trained staff in one facility/location capable of even beginning to change the culture and practice.

Another area which has a very high and continued demand for CMEs is neonatal resuscitation training in response to high levels of neonatal mortality and stillbirth. As managers of the SVP we are constantly asked to provide such training. However, the experience of professional volunteers working in the facilities indicates a very poor level of skills application. In one case quite shortly after the completion of this training, a baby was born in the facility requiring resuscitation; local staff were unwilling to use their skills and insisted that SVP volunteers resuscitated the baby. This may reflect a perfectly understandable lack of confidence in their new clinical skills - it is one thing to resuscitate a mannequin in a training room and quite another to resuscitate a newborn baby. But this experience 
Table 3.3 Improved outcomes as a result of short courses

\begin{tabular}{lcl}
\hline Indicator & Baseline & Endline \\
\hline Partograph use & $4 \%$ (Jan 2012) & $79 \%($ Feb. 2015) \\
Active management of third stage of labour & $5 \%$ (Jan 2012) & $97 \%$ (Feb 2015) \\
Screening for pregnancy-induced hypertension & $48 \%($ Nov 2013) & $68 \%($ Feb 2015) \\
Successful resuscitation of asphyxiated babies & $67 \%($ Aug 2013) & $81 \%($ Feb 2015) \\
Provision of essential newborn care services & $1 \%$ (Jan 2012) & $85 \%$ (Feb 2015) \\
Family planning counselling & $40 \%$ (Jan 2012) & $91 \%$ (March 2015) \\
Family planning update & $10 \%(\operatorname{Jan} 2012)$ & $67 \%$ (March 2015)
\end{tabular}

Source: http://savingmothersgivinglife.org/our-work/reports.aspx

is replicated across all facilities even where training has attempted to achieve a level of 'saturation', suggesting that training in many cases is failing to translate into behaviour change (implementation).

As we noted in Chapter 1, funding bodies and projects are understandably keen to 'prove their concept' and this results in the development of metrics that appear to suggest significant attributable outcomes. A recent blog by a senior Ugandan actor in an ambitious and highly lucrative USAID project (Saving Mothers Giving Life) is an extreme example of this. The short report claims overwhelming successes arising from what he refers to as 'high-impact interventions over a short period of time'. This statement is backed up by a table providing quantitative indicators of success (Table 3.3):

These figures purport to relate to public health facilities in the Fort Portal region - an area we are very familiar with. Sadly they bear little relation to the reality on the ground and create an entirely false impression that short course training immediately impact systems.

A far more cautious and in-depth review of another major training intervention funded this time by the UK's Department for International Development (DFID) of its 'Making it Happen' programme (Phase 2) presents data showing that between 2012 and 2015, 17,000 health workers were trained in emergency obstetric and neonatal care across 11 African countries. ${ }^{7}$ The intense EmONC course is 6 days long and is delivered by multi-disciplinary teams using 'expert' volunteers. It aims to train a critical mass of $80 \%$ of healthcare providers in the facilities involved. The programme has cost $£ 18$ million to date. However, the 2015 Annual Review refers to the 'difficulties of measuring the impact' and, on the basis of the data they could collect, reports that three out of 
the four outcomes are 'off track'. It is important to note that the report presents evidence of some post-course improvements but these are often quite minimal. Outcome 1, for example, aimed to increase the number of women attending participating healthcare centres for delivery by $20 \%$ over 12 months: in practice, the improvement was around $1.5 \%$. And, Outcome Indicator 4: 'to reduce facility level newborn deaths by an average of $15 \%$ ' seemed to have failed with a reported increase of $44 \%$ in just under half of the facilities. The point here is not to criticise this programme but to raise fundamental questions about the efficacy of short-term CME-style training and also the quite impossible metrics that funded projects are having to try to align themselves with. The Making it Happen report reflects on the need for 'qualitative research' to help provide a 'better understanding of the causes of deaths' and the impacts of the programme. It also suggests that more work needs to be done with governments to 'stabilise human resource situations' and 'reduce staff rotations', which undermine both the efficacy of training and the ability to control for and measure outcomes.

The effect of staff rotation ('turnover or transfer of trained health workers in the overseas institution') on the ability to embed and/or evaluate impacts is so well known that it is explicitly identified as a 'barrier to change' that applicants are required to respond to when making an application for THET funding (THET 2015: para 4.4).

In the SVP context, a strong pattern has emerged of rotation of Ugandan staff either during or immediately after training and mentoring interventions. In some cases, Ugandan healthcare workers have been transferred from the facilities or wards where volunteers have been working as soon as training has taken place and in the absence of any communication to either UK partners or the Ugandan health workers. Clearly, it is the prerogative of Ugandan authorities to manage their staff appropriately and this will imply moving staff at times. However, this practice has taken place following the return to Uganda of health workers supported for training in the UK under the British Commonwealth Professional Fellowship scheme and represents a significant loss of UK resource and disrespect for the scheme. In many cases, the re-deployment of Ugandan staff who have established strong relationships with volunteers and health partnerships appears to represent a deliberate attempt to break relationships and 'punish' Ugandan health workers. This may reflect a perception, once again built up through many years of voluntarism, that Health Partnerships are privately remunerating local health workers. This has a 
very damaging effect on health partnerships, on volunteer-health worker relationships and represents a highly inefficient way of deploying development resource.

Schaaf and Freedman document the effects of what they term 'Mission Inconsistent' (MI) posting in low-resource settings. They argue that the focus on 'calculating [skills] deficits and organising training' (2015: 1) has overlooked challenges that 'those who work on the ground' often see. And one of these is the effect of 'posting' or staff transfers on motivation. They identify two common scenarios. In the first place, health workers themselves may 'employ clientelism or bribery or obtain a post in a desirable area' (perhaps a location where they can optimise income from commodity sales or bribes). De Zwart (2000) refers to these as 'earning centres'. On the other hand, managers (as in the case earlier) may 'express displeasure' with a healthcare worker by re-locating them. This displeasure may be tripped by a perception that the health worker has established close (and potentially fruitful) relationships with volunteers or NGOs. Schaaf and Freedman conclude that in-depth qualitative methods are necessary to generate an 'emic understanding' of how posting and staff transfers are negotiated:

Emic research will inform efforts that can work with the grain, by understanding the nuances of a particular social, political and economic context, and identifying avenues for meaningful change. (2013:7)

Consideration should be given to the idea of developing firm contracts with managers perhaps through Memoranda of Understanding (MOUs) or local 'Human Resource Compacts' with agreements to 'bond' or retain trained staff for periods of 3-5 years to enable the training to embed and project objectives to be achieved. Breach of these conditions should be responded to accordingly through a reporting mechanism to the Ministry of Health (via the Uganda-UK Health Workforce Alliance) and withdrawal of Health Partnership support. This is an area we are currently working to implement in the Fort Portal Health Partnership.

Intense off-the-job training via CMEs, in isolation, appears to be quite effective in terms of conveying explicit clinical or technical information in the short term at least. There is minimal evidence to show that this knowledge is retained or, of greater concern, utilised. And, our observational research within the frame of the SVP would suggest that there is 
little evidence that this is taking place. Of course, it is also difficult systematically to argue the opposite - that it is not taking place. What we are aware of is that when CMEs are embedded within on-going mentoring and reinforced through co-working during SVP volunteer stays the chances of knowledge translation and application are much greater. Some of the factors contributing to this process are as follows:

First, exposure to a new clinical skill may not lead to implementation because the knowledge is partial, not fully understood or because the participant needs supervision/support to enable them to use the skill for the first time. Confidence in actually using a skill is often built up over time in a supportive environment.

Secondly, from a knowledge perspective, the transmission of explicit clinical skills may not in itself be enough: explicit skills may need to be nurtured 'on-the-job' in combination with more highly contextualised tacit skills to begin to achieve implementation. And this tacit knowledge may be valuable both for the Ugandan health workers and the UK professional volunteers seeking to improve skills. Foreign fly-in-fly-out 'experts' are unlikely to possess the kinds of in-depth tacit knowledge that enables them to understand the healthcare context within which the 'new' skills could be utilised. Ironically, they are likely to be seen by many local actors as naïve and lacking in contextualised local knowledge.

\section{From Training to Mentoring (Or Combining Training with Mentoring)}

All you do is train, train train (UHW)

This comment was made to Professor Ackers during a review of the placement of SVP volunteers by a senior Ugandan midwife. She went on to urge the SVP to deploy volunteers in roles that 'enabled them to work alongside us - to work together'. Her point was well made and perfectly understandable in a small health centre IV facility that had so few local staff it could barely function and an in-charge doctor who refused to do any clinical work at all. In practice, the depletion of local midwifery staff exacerbated by the removal of doctors prior to completion of their fellowships in the UK (as a form of punishment) by the local District Health Officer eventually meant that co-presence was unworkable and we regretfully made the decision to withdraw. 
Chapter 1 introduced the principle of co-presence as a necessary but not sufficient basis for effective knowledge exchange. Of course, co-presence is relatively easy to achieve in educational programmes and CMEs - to the extent that the co-presence is with students and learners. We would argue however that co-presence at this level requires also that professional volunteers are co-present with Ugandan trainers and educators - in a coteaching format. This was the approach taken in the SVP EmONC course where Ugandan clinicians returning from advanced training at the Liverpool School of Tropical Medicine worked alongside their UK peers. Unfortunately, on the next planned delivery of the programme the Ugandan trainers demanded a much higher level of remuneration than previously paid (now that they had UK certificates!) and failed to join the training team. Their skills had become commodified and they were using them as an income-generation device, having set up their own training NGO rather than in their clinical work.

In a clinical context, co-presence is more difficult to achieve but absolutely essential to knowledge transfer and, more specifically, to the processes of knowledge translation and utilisation. Professional volunteers with clinical skills acquired from the UK need these relationships to be able to apply these skills in the very different cultural and resource context they find themselves in. And their Ugandan peers need this level of support and mentoring to build confidence to translate and apply skills learnt in CMEs. Building on our previous evaluation experience, the SVP began to focus on mentoring and coworking as the primary mechanism for knowledge translation and application.

\section{Mentoring, Co-working and Knowledge Translation}

Our awareness of the limited impact and externality effects associated with formal 'off-the-job' CME training interventions combined with the risks and systems damage caused by labour substitution encouraged us to promote a mentoring approach to knowledge mobilisation. We have used the concept of mentoring rather than supervision to capture the bi-lateral co-learning and knowledge exchange quality of these processes. The idea has been to place professional volunteers, as knowledge intermediaries, into situations where they can work alongside their peers to promote learning-through-doing. This may both play a role in identifying the need for formal intense off-the-job training, perhaps 
creating opportunities for short-term 'fly in' clinical support or act as a critical follow on from this.

We noted earlier on the tendency for programme audit or internal evaluation requirements to skew interventions framed around the quest for metrics. Whilst we know that this approach to knowledge mobilisation is far more effective and less damaging than traditional approaches, it presents significant challenges in terms of project reporting (and perceived impact/success). Under pressure from THET to deliver regular quantitative data on training numbers, we invited one of the SVP volunteers to try to estimate her mentoring encounters. In the following excerpt, the volunteer (a British obstetrician) describes one working day and the kinds of mentoring she was engaged in:

I teach all day every day and each session runs into others. If I give you an example of my day today you will see what I mean.

In my ward round with 2 interns and a midwife the first patient was in obstructed labour at fully dilated. I discussed with them the use of syntocinon and how it should not be used in multips for augmentation. I discussed the indications and contraindications for instrumental delivery. I discussed with them the value of being able to ascertain positions and the likely causes of obstructed labour in a multip. The second patient was an IUFD at $35 \mathrm{ks}$ with a genital ulcer. We discussed the causes of genital ulcers, the investigations and treatments. We discussed the options for delivery including induction and the different methods of induction that can be employed. We talked about the riskes of doing an induction in a patient with a previous CS. $2^{\text {nd }}$ patient cord proplapse with IUFD; we discussed management of cord prolapse and the best method of delivery in cord prolapse with IUFD.

The third patient had premature rupture of membranes. We discussed the role of augmentation and antibiotics. The fourth patient had preeclampsia and was undergoing induction. We went through the signs and symptons of PET and the value of assessing them. We discussed the fact that syntocinon in the presence of intact membranes can cause amniotic fluid embolus. Would this count as teaching $2^{\prime} 3$ people on 11 topics?

I then assisted the intern at c-section. I talked him through what to do in a transverse section. He struggled to deliver the head so I took over. We then discussed techniques to help in the delivery of the head. I then assisted the intern at a second c-section. This one had a previous caesarean section and we discussed the likely complications and how to avoid them. We did the c-section and again I assisted with the delivery of the head. We talked 
about the lack of tone and the deformities that made it difficult. I then went with an intern and a midwife to labour suite to see another woman with three previous $\mathrm{c}$-sections and 4 normal vaginal deliveries and a malpresentation. We discussed risk of scar rupture and risk of cord prolapse. I did a caesarean section for a woman with 3 previous $\mathrm{c}$-sections and a malposition. Prior to the c-section I took the intern through the delivery of breech at $\mathrm{c}$-section (and at vaginal delivery). During the caesarean I discussed what I was doing and why. At all three caesarean sections I went through the theatre check list with the interns, including checking of the $\mathrm{FH}$ where appropriate. I also discussed with them both the importance of counting swabs afterwards and initiated this with each case. I did this in front of all the theatre staff and the anaesthetist, although not directly talking to them.

In the breaks between theatre I discussed a topic that we talked of yesterday, the exteriorisation of the uterus and closure of the peritoneum. I brought along some papers and evidence including Cochran reviews for this and we discussed it briefly. I encouraged them to go and read the papers then we would talk again.

At the end of the day one of the interns discussed with me the value of vaginal birth following caesarean section. We discussed this and the value of pelvimetry for about $30-40$ minutes.

As individual teaching events to individuals this day I'm guessing in the hundreds. This is just one day, and it is not unusual. There is no way I could count individual teaching events in a month; it will be in the thousands. I could count hours involved in teaching, but it will probably be about $6-8$ hours a day. I try to do no clinical activities without it being an educational exercise.

This discussion illustrates the complex and significant contribution the volunteer is making on a daily basis to knowledge mobilisation. However, it also shows how the pressure for evaluation metrics is tending to shape interventions and mentoring is a case in point. In many respects,

Q - Our approach is to mentor health workers. How should we count the number of trainees? A - Please report to number of health workers you have mentored. You may need to ask your volunteers to tally them over the course of each reporting period.

$Q$ - We train health workers on the job. How should we count the number of trainees?

A - Please report to number of health workers you have trained on-the-job. You may need to ask your volunteers to tally them over the course of each reporting period.

If you train the health workers in two distinct areas or topics, we would like you to count the health workers twice even though they are the same people. If you spread training on a single topic over a long period then please do not count the health workers twice.

Fig. 3.2 THET frequently asked questions (Source: THET 2016) 
it defies quantitative measurement. In recent months, THET has responded to this 'challenge' by producing guidelines on how to measure mentoring (Fig. 3.2):

We do not believe it is possible or methodologically appropriate to even attempt to capture data in this way. Certainly the results will massively increase the volume of 'encounters', but how should we interpret these? Fully capturing the effects over time and space of knowledge mobilisation through co-presence demands a quite different, more ethnographic, approach. Our experience with SVP volunteers suggests that mentoring is a far more successful mechanism for volunteers acting as knowledge intermediaries, providing a fruitful environment for mutual learning and doing. Having said that, we have to report the fact that in most cases the impact of even these interventions can be relatively short lived and rarely if ever extends beyond the period of co-presence. Where changes in behaviour are observed, there is typically a rapid time decay in implementation reverting to prior behaviour very quickly once co-presence ceases. One of the British Doctors involved in a Health Partnership described this experience as 'dipping your finger in a pool of water and then taking it out and watching the ripples disappear'. (Ackers 2014, Volunteer and Health Worker Relationships Policy Report, p. 2).

\section{Conclusions}

Chapter 3 has built on the concerns expressed in Chapter 2 about labour substitution and its impact on Ugandan human resource systems. The chapter has exposed some of the problems and externality effects associated with the particular form of training that many, if not most, foreign organisations and professional volunteers are engaged in; namely, short courses or forms of continuing professional development. There is undoubtedly a need for continuing medical education as many of those health workers that volunteers directly encounter on the wards will be in need of additional training and support. However, the approach to CME provision by external bodies has developed into a culture in which training has been commodified - not to the extent that it is in the UK where the trainee has often to pay very high fees in order to receive training - but rather where the trainer is expected to pay learners for the privilege. In practice, this means that many of those people attending training are not intrinsically interested in the 
skills and many of those health workers who really need training are not given the opportunity to attend. Training also becomes one of a range of factors reducing (quite significantly in many cases) the amount of time more senior health workers spend in clinical work. Many doctors will never undertake clinical work in their public sector roles.

The kind of training that takes place off-site in CMEs may be effective in terms of transferring explicit technical or clinical skills but is unlikely in most cases to transmit the complex knowledge combinations essential to the effective translation and operationalisation of knowledge. When used in combination with mentoring and co-presence on the job, there is greater evidence of impact and skills utilisation and some real progress can be made. However, even in these cases, systems seem to be able to slip back as quickly as they progress once volunteer presence ceases. Why change is so short lived and so conditional on volunteer presence is a conundrum challenging many health partnerships. The 'exit strategies' that funding bodies and development organisations talk so much about appear hard to achieve in practice. Chapter 4 explores some of the reasons for the short-lived quality of systems change.

\section{Notes}

1. This is technically illegal but commonplace as many short-term volunteers operating outside the frame of structured (and funded) programmes are unwilling or do not understand these quite bureaucratic, time-consuming and expensive processes.

2. For details of the THET-funded bio-medical engineering project, see www. Salford and/or http://www.knowledge4change.org.uk/.

3. The SVP does not pay per diems as such but will cover essential expenses where necessary.

4. It is very hard to organise events without such conflicts arising as there is a received wisdom locally that it is best not to encourage NGOs to cooperate as this may reduce personal opportunities.

5. This is right and proper and the SVP has taken every step to build such relationships and ensure that senior managers are aware of and in support of interventions. It is however important to point out that in some cases local managers use this 'opportunity' to extract personal financial inducements; a kind of personal top-slice from all foreign NGOs as a price for permitting the engagement. This was another reason why the SVP, as a project, has withdrawn from engagement in Wakiso District. 
6. Indeed, there were so many Ugandan obstetricians at this event which was followed by another event in Canada that some regional referral hospitals had to close obstetric theatres.

7. Annual Review 2015. The programme is implemented by the Centre for Maternal and Newborn Health at the Liverpool School of Tropical Medicine (CMNH-LSTM).

Open Access This chapter is distributed under the terms of the Creative Commons Attribution 4.0 International License (http://creativecommons.org/ licenses $/$ by $/ 4.0 /$ ), which permits use, duplication, adaptation, distribution, and reproduction in any medium or format, as long as you give appropriate credit to the original author(s) and the source, a link is provided to the Creative Commons license, and any changes made are indicated.

The images or other third party material in this book are included in the work's Creative Commons license, unless indicated otherwise in the credit line; if such material is not included in the work's Creative Commons license and the respective action is not permitted by statutory regulation, users will need to obtain permission from the license holder to duplicate, adapt or reproduce the material.

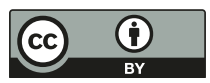

